\title{
HACER BIEN LAS COSAS CUANDO SE NECESITAN, SIN PRISAS PERO SIN PAUSAS, UNA VERSIÓN ESPAÑOLA DEL LEAN SIX SIGMA
}

\section{DO THE RIGHT THINGS WHEN IT IS NEEDED, UNHURRIED BUT STEADILY (A SPANISH VERSION OF LEAN SIX SIGMA)}

Francisco José Senent Torices ${ }^{1}$ Víctor Gisbert Soler ${ }^{2}$

1. Master Universitario de Ingeniería de la Organización y Logística por la Universidad Politécnica de Valencia (España). E-mail: joseto@epsa.upv.es

2. Doctor Ingeniero Industrial. Departamento de Estadística e Investigación Operativa Aplicadas y Calidad en la Universidad Politécnica de Valencia (España). E-mail: vgisber@eoi.upv.es

\section{Citación sugerida:}

Senent Torices, F.J. y Gisbert Soler, V. (2017). Hacer bien las cosas cuando se necesitan, sin prisas pero sin pausas, una versión española del Lean Six Sigma. 3C Empresa, investigación y pensamiento crítico, 6(1), 54-62. DOI: <http://dx.doi.org/10.17993/3cemp.2017.060129.54-62/>. 


\section{RESUMEN}

A estas alturas de la crisis económica que ha afectado a todas las economías mundiales, en España con mayor profundidad que en otros países, nos ha hecho plantearnos si nuestro sistema productivo como país es competitivo o eficiente en comparación con los existentes en otro países de nuestro mismo nivel. La respuesta creo que está clara, NO.

De una manera u otra muchas empresas de España, PYMES la mayoría, han buscado en el ahorro interno, la mejor manera de convertirse en empresas eficientes y productivas. Algunas por desgracia, tomaron el camino de la reducción de personal de manera directa, y otras optaron por analizarse internamente para ver por dónde estaban "perdiendo dinero", vía que les ha permitido balancear su margen hasta convertirlo en positivo o generar un mayor margen en su actividad.

Son varios los métodos o filosofías que se han implantado en España como herramientas para optimizar los procesos productivos de las empresas, Lean Manufacturing y Six Sigma, en mayor grado el primero que el segundo. De igual manera y como casos excepcional se ha intentado implantar una filosofía mixta, el Lean Six Sigma.

¿Pero están preparadas las PYMES españolas en implantar estas filosofías o metodologías?, ¿funciona el Lean Six Sigma?, ¿se ha implantado el Lean Six Sigma en otros países distintos que España?

\section{ABSTRACT}

At this stage of the economic crisis that has affected all global economies, in Spain more deeply than in other countries, it has made us ask ourselves if our production system as a country is competitive or efficient compared with other countries in our same level. I think the answer is clear, NO.

In one way or another many companies in Spain, most SMEs have sought in domestic savings, the best way to become efficient and productive enterprises. Some unfortunately, took the path of retrenchment directly, and others chose to be analyzed internally to see where they were "losing money". This way has enabled them to balance their margin to make positive or generate a greater margin their activity.

There are several methods that have been implemented in Spain as a tool to optimize production processes in companies, Lean Manufacturing and Six Sigma, and in a greater degree the first than the second one. Similarly, and as exceptional cases it has tried to implement a mixed philosophy, Lean Six Sigma.

But are Spanish SMEs prepared to implement these philosophies or methodologies?, Lean Six Sigma works?, has been implemented the Lean Six Sigma in other countries different to Spain? 


\section{PALABRAS CLAVE}

Lean Manufacturing, Seis Sigma, Pyme, competitividad, valor añadido

\section{KEY WORDS}

Lean Manufacturing, Six Sigma, Pyme, competitiveness, value added. 
En los últimos años se han realizado esfuerzos organizados destinados a conseguir la mejora continua, muchos de ellos conocidos por su terminología inglesa o hasta japonesa. Todos ellos, hasta los que se han quedado por el camino o han sido absorbidos, tenían conceptos importantes, herramientas y métodos útiles.

Por desgracia también tenían fallos en sus filosofías que se pusieron de manifiesto en la implementación de los mismos, y en alguna ocasión, en las mismas hipótesis en las que estaban basados.

Uno de ellos, el Lean Six Sigma, ha tenido, en mi opinión igual que el resto, una gran dificultad para ser implantados en el tejido empresarial español, constituido principalmente por PYME's.

Han sido varios autores los que han manifestado métodos de implantación del Lean Six Sigma en PYME's y los que han sacado a la luz sus virtudes y defectos. De igual manera han propuesto posteriormente sus metodologías.

\section{METODOLOGÍA}

Lean Six Sigma, metodología o enfoque que combina el Lean Manufacturing y el Six Sigma, que permite que las organizaciones consigan ganancias sistemáticas que proporcionen una mejora en el resultado final de la misma.

Primero de todos y de la manera más resumida posible vamos a definir cada una de las metodologías que se han fusionado para crear el sistema Lean Six Sigma, en adelante (LSS), según Dee Jacob, Suzan Bergland y Jeff Cox (2010), las metodologías son;

- Lean Manufacturing se fundamenta en base a conceptos establecidos por el sistema de producción de Toyota, TPS, y gira en torno a la eliminación del desperdicio en todas sus formas.

- Six Sigma surge a partir de la gestión de la calidad total y de otros métodos de mejora de la calidad, y su gran ventaja es que reduce la variación.

Ahora bien, ¿qué tienen de común o de ventajoso cada uno de los métodos para poder fusionarse y formar el LSS?, según Felizzola y Luna (2013), cada uno de estos enfoques hace un gran aporte a la mejora continua desde diferente frentes [3];

- La disminución de defectos de forma estructurada en el caso del Six Sigma.

- Y el mejoramiento de los flujos de procesos e información por medio del Lean Manufacturing.

Estas características permiten la integración del Lean Manufacturing y del Six Sigma para constituir el LSS. 


\section{IMPLANTACIÓN DEL LEAN SIX SIGMA EN LAS EMPRESAS}

Una vez definido el LSS y verificar de las bondades que aportaría esta filosofía a las empresas ahora queda implantar la filosofía en las mismas, las entidades de la sociedad que más se pueden beneficiar.

En España el 99,99\% de las empresas son Pymes, por tanto el enfoque que se le tiene que dar a la implantación de cualquier sistema o metodología de trabajo es un enfoque de Pymes. Las Pymes se caracterizan en su lado negativo por:

- La falta o pérdida del compromiso de la dirección en algún proyecto que se inicie

- La no existencia de cultura organizacional de la empresa

- El choque del proyecto con la estrategia de la organización

- La no formación

- La falta de capacidades y competencias para la gestión y ejecución del proyecto

- La capacidad financiera

Por lo tanto, para evitar caer en los aspectos negativos que pueden afectar en la implantación del LSS en una empresa PYME se debe de buscar una metodología que sea:

- Con objetivos, pequeños, a corto plazo para mantener vivo el espíritu de la implantación

- Fácil de integrarse en el día a día de la empresa

- Respetuosa con la estrategia de la empresa siempre que estas puedan ser compatibles

- Fácil de asimilar

- Y no costosa.

\section{$\underline{\text { Revisión de la literatura acerca del LSS }}$}

No sería muy inteligente ignorar lo ya existente sobre metodologías de implantación de LSS por lo que vamos a analizar los mismos.

Thomas, Barton \& Chuke Okafor proponen un modelo para implantar de forma integrada el Lean Manufacturing y el Six Sigma de manera integrada. En una PYME se obtuvieron resultados positivos en calidad, costes y tiempo de entrega [1].

Chakravorty desarrolla un modelo compuesto por 6 etapas, análisis, equipos de mejora, herramientas de mejora, mapeo organización e identificar oportunidades, desarrollo de plan detallado y por último implantación [7].

Knowlesa, Whickreb, Heraldez \& Del Campo, desarrolla un modelo de implantación de Six Sigma formado por dos grandes ciclos, el 10 se ocupa de aspectos estratégicos, definir

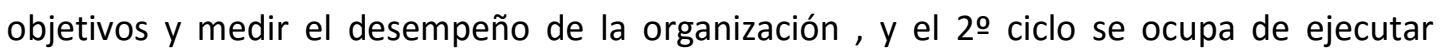
proyecto Six Sigma utilizando DMAIC [2].

Kumar, Antony \& Tiwari, proponen un modelo que está compuesto por 12 pasos divididos en 5 fases. Este modelo facilita una guía paso a paso para que se pueda implementar, desde la definición hasta el sostenimiento a largo plazo [4]. 
Gnanaraj, Devadasan, Murugesh y Sreenivasa, desarrollan un modelo denominado DOLADMAICS, acrónimo de Deficiency, Overcoming, Lean, Anchorage, Define, Measure, Analize, Improve, Control, Stabilise [6].

Al hacer lectura solamente del resumen de los artículos citados se tiene una sensación de que la implantación no será nada fácil de compaginar con el día a día de cualquier PYME, empresas que en ocasiones centran sus esfuerzos en la supervivencia, una metodología de 12 pasos puede convertirse en una tarea ardua de ejecutar.

Citamos de manera especial el modelo propuesto por Felizzola y Luna, el cual se fundamenta en 4 fases, siendo la fase inicial, preparación, la fase más intensa en cuanto a las debilidades de las PYME's citadas anteriormente.

De la fase de preparación, fase 1, destacar que se busca:

- Compromiso de la dirección

- Alineación estratégica

- Enfoque al cliente

- Formación en lean six sigma

- Estandarización de procesos

- Cultura de medición

Poder empezar una metodología de trabajo con los fundamentos indicados en los 6 puntos anteriores, puntos de la fase 1 , es avanzar de manera considerable ante cualquier reto, compromiso de la dirección principalmente.

Tan poco puedo dejar pasar por alto la metodología desarrollada por Mantilla Celis \& Sánchez García para implantar en este caso LSS específico para el sector de la Logística [5].

Del modelo resaltar las 5 fases que lo configuran, fase de Definición, de Medición, de Análisis, de Mejoramiento y de Control. Destacar la capacidad de claridad que se detalla el mismo, señalando en cada paso de cada fase;

- Las interrogantes que nos encontraremos

- Las herramientas que podremos utilizar para solucionar las dudas del interrogante

- Los resultados obtenidos al emplear las herramientas citadas

- El objetivo alcanzado de cada una de las fases

Tan poco podríamos dejar de señalar que de las dos metodologías que hemos analizado con más profundidad el número de pasos o fases para implantar las mismas son elevados, convirtiéndolas en una travesía por el desierto.

\section{Propuesta}

Para no caer en los errores que hemos resaltado de los otros métodos, buscaríamos reducir al máximo los pasos de implantación de la metodología. 


\section{Fase de previa}

\section{¿El dueño de la empresa entiende la finalidad de la implantación de la metodología?}

En ocasiones, con un análisis previo de manera directa con el propietario de la PYME resolvemos la cuestión planteada. Por desgracia podemos encontrar a consultores que busquen iniciar y prolongar la implantación de la metodología siendo conocedor de que el mismo va a fracasar por la no implicación de la propiedad.

¿El dueño de la empresa asume que la implantación será un proceso costoso económicamente, sacrificará los recursos humanos de la empresa y alterará el día a día de la empresa?

Dinero, conflictos y sacrificio, son adjetivos que podríamos enumerar, recursos que se consumirán, situaciones que se darán y esfuerzos que se necesitaran.

¿La cultura de la empresa y la capacidad de los trabajadores de la misma permitirán implantar la metodología?

En ocasiones pedimos a una empresa que no ha sido capaz de implantar una ISO 9001 que sea capaz de obrar un milagro implantando un modelo Lean Manufacturing o Six Sigma.

Tan pronto una de estas preguntas tenga respuesta NO, deberíamos de detener el proceso de implantación. Evita problemas generándolos tú mismo.

\section{Fase de definición}

¿Qué quieren nuestros clientes?

Conocimiento de las expectativas y requerimiento del cliente de nuestros productos y de nuestra empresa. (Análisis KANO)

\section{¿Puedo repetir de manera constante lo que me pide mi cliente?}

Como empresa productiva puedo repetir las cualidades que mi cliente ha detallado sobre mis productos de manera que no difieran la misma. (Análisis gráfico XR - XS)

\section{¿Cómo puedo llegar a lo que quiere mi cliente de manera eficiente?}

Conozco mi empresa de manera eficiente desde la recepción del feedback del cliente que me debe de guiar para diseñar producto/servicio hasta la entrega de su producto. (Value Stream Mapping)

Una vez tenga claro a dónde quiere llegar, que es capaz de hacerlo y que sabe hacerlo eficientemente, ADELANTE, IMPLANTA EL LSSiiii

\section{Fase de análisis. (Parte de Six Sigma)}

Se debe de seleccionar las herramientas más adecuadas en función de la naturaleza del problema.

Podrimos enumerar miles de herramientas pero señalaremos aquellas que son más sencilla de utilizar: 
Diagrama de Pareto, Grafico de control, Diagrama causa - efecto, Anova, Regresión Lineal, etc.

Estas herramientas nos permitirán detectar los errores graves y las variaciones que se dan en nuestro proceso productivo

\section{Fase de mejora (parte de Lean Manufacturing)}

Se debe de seleccionar las herramientas más adecuadas en función de la naturaleza del problema.

Podrimos enumerar miles de herramientas pero señalaremos aquellas que son más sencilla de utilizar:

5'S, Control Visual, TPM, SMED, Poka Yoke, Estandarización, etc.

Aplicando 4 de ellas aumentaríamos nuestra eficiencia eliminando lo que NO da VALOR, y que nuestro día a día sea aportar valor.

\section{Fase Final}

Verificar que hemos mejorado, comparando el antes / el después. Ojo y no olvidar el cliente, preguntar si ha notado mejora, menos coste, menos tiempo de entrega, más garantías como usuario, etc.

\section{CONCLUSIONES}

En el presente artículo se han analizado diferentes métodos de implantación del Lean Six en PYME's, aplicables o no dependiendo de la actividad y características de la organización en la que se quieran implementar.

Se han analizado metodologías que permiten implantar de forma integrada Lean Manufacturing y Six Sigma, basadas en diferentes ciclos, normalmente dos, o etapas, de seis hasta doce.

En todas las metodologías y propuestas de metodologías se concluye en la dificultad de su implementación en la pyme.

A pesar de las diferentes posibilidades y enfoques se concluye en que para no caer en los errores que de los diversos métodos analizados, quizás sería una buena estrategia el reducir al máximo los pasos de implantación, quedándonos en cinco fases: previa, definición, análisis (six sigma), mejora (lean manufacturing) y una final de verificación. 


\section{REFERENCIAS BIBLIOGRÁFICAS}

[1] A. Thomas, R. Barton and C. Chuke-Okafor. (2008). Applying lean six sigma in a small engineering company - a model for change. Journal of Manufacturing Technology Management, 20(1), 113-129. ISSN: 1741-038X. DOI:10.1108/17410380910925433.

[2] G. Knowles, L. Whicker, J.H. Femat and F.D.C. Canales. (2005). A conceptual model for the application of Six Sigma methodologies to supply chain improvement. International Journal of Logistics Research and Applications, 8(1), 51-65. ISSN: 13675567. DOI: 10.1080/13675560500067459.

[3] Heriberto Felizzola J, Carmenza Luna A. (2014). Lean Six Sigma en pequeñas y medianas empresas: un enfoque metodológico. Ingeniare. Revista Chilena de ingeniería, 22(2), 236-277.

[4] M. Kumar, J. Antony and M.K. Tiwari. (2011). Six Sigma implementation framework for SMEs - a roadmap to manage and sustain the change. International Journal of Production Research, 49(18), 5449-5467. ISSN: 0020-7543. DOI: 10.1080/00207543.2011.563836.

[5] Olga L. Mantilla Celis, Jose M. Sachez. (n.d). Modelo tecnológico para el desarrollo de proyectos Logisticos usando Lean Six Sigma. Estudios Generales, 28(124), 23-24.

[6] S.M. Gnanaraj, S.R. Devadasan, R. Murugesh y C.G. Sreenivasa. (2012). Sensitisation of SMEs towards the implementation of Lean Six Sigma - an initialisation in a cylinder frames manufacturing Indian SME. Production Planning \& Control, 23(8), 599- 608. ISSN: 0953-7287. DOI: 10.1080/09537287.2011.572091.

[7] S.S. Chakravorty. (2009). Six Sigma programs: An implementation model. International Journal of Production Economics, 119(1), 1-16. ISSN: 0925-5273. DOI: 10.1016/j.ijpe.2009.01.003. 